This implies $\operatorname{dom} P$ is a subset of $Q$, and since $\max R$ is empty, dom $P=Q$. Thus $P \cup M$ is a solution of $(S,>)$.

\title{
REFERENCES
}

1. D. Gillies, Some theorems on n-person games, Thesis, Princeton, 1953.

2. D. König, Uber Graphen und ihre Anwendung auf Determinantentheorie und Mengenlehre, Math. Ann. vol. 77 (1916) pp. 453-465.

3. J. von Neumann and O. Morgenstern, Theory of games and economic behavior, 2d ed., Princeton, 1947.

4. M. Richardson, On weakly ordered systems, Bull. Amer. Math. Soc. vol. 52 (1946) pp. 113-116.

TULANE UNIVERSITY AND

The Institute for Advanced Study

\section{SUBALGEBRAS OF AN ALGEBRA WITH A SINGLE GENERATOR ARE FINITELY GENERATED}

DAVID GALE

In order to prove the assertion above it clearly suffices to consider only the free algebra on one generator and hence the polynomials in one variable over a field $F$. The fact that the sub-algebras of $F[x]$ are all finitely generated is perhaps not surprising, but the author has not been able to discover any proof of it in the literature. (Incidentally, the corresponding statement with the field $F$ replaced by the ring of integers is not true, for the ring of all integral polynomials with even coefficients is clearly not finitely generated.) The proof, though quite simple, would seem to place the problem outside the category of elementary exercises since the solution, at least as presented here, uses some "theory," namely the ever useful facts about modules over principal ideal rings.

We shall prove a somewhat stronger result, namely

Theorem. Let $A \neq F$ be a subalgebra of $F[x]$ and let $n$ be the degree of the polynomial of smallest positive degree in $A$. Then $A$ can be generated by a set of not more than $n+1$ elements.

Proof. We consider first the case $F \subset A$. Let $p$ be a polynomial in $A$ of degree $n$, and let $F[p]$ denote the algebra generated by $p$ and $F$ in $F[x]$. We assert that $F[x]$ is a module over $F[p]$ with generators $1, x, \cdots, x^{n-1}$, for if $q \in F[x]$ we show by induction on the degree of

Received by the editors August 11, 1956. 
$q$ that $q=\sum_{i=0}^{n-1} r_{i} x^{i}$, where $r_{i} \in F[p]$. Namely, by the Euclidean algorithm $q=a p+\sum_{i=0}^{n-1} \alpha_{i} x^{i}, \alpha_{i} \in F$, and by induction hypothesis $a=\sum_{i=0}^{n-1} r_{i} x^{i}, r_{i} \in F[p]$, so $q=\sum_{i=0}^{n-1}\left(r_{i} p+\alpha_{i}\right) x^{i}$, proving the assertion.

Now $F[p]$, being an algebra on one generator over $F$, is a homomorphic image of $F[x]$ (in fact, isomorphic in this case) so, in particular, $F[p]$ is a principal ideal ring. We have just shown that $F[x]$ is a module on $n$ generators over $F[p]$, and, since $A$ is a $F[p]$-submodule of $F[x]$, it follows from a basic theorem on modules over a principal ideal ring (see, for instance, Bourbaki, Livre II, Chapitre VII, §3, Proposition 1, p. 82) that $A$ has a set of generators, $g_{1}, \cdots, g_{m}$ with $m \leqq n$. Thus, every element of $A$ can be written in the form $\sum_{i=1}^{m} r_{i} g_{i}$ where $r_{i} \in F[p]$, and hence $A$ is generated by the elements $p, g_{1}, \cdots$, $g_{m}$.

If $A$ does not contain $F$, let $\bar{A}$ be the algebra generated by $A$ and $F$. We have just shown that $\bar{A}$ has a set of not more than $n+1$ generators $\bar{f}_{1}, \cdots, \bar{f}_{k}$. Now $\bar{A}$ is a vector space direct sum of $A$ and $F$, hence for each $i, \bar{f}_{i}=f_{i}+\alpha_{i}$ uniquely, where $f_{i} \in A, \alpha_{i} \in F$. If $q \in A$ then $q$ is expressible as a polynomial $P$ in the $\bar{f}_{i}, q=P\left(\bar{f}_{1}, \cdots, \bar{f}_{k}\right)$ $=P\left(f_{1}+\alpha_{1}, \cdots, f_{k}+\alpha_{k}\right)=P^{\prime}\left(f_{1}, \cdots, f_{k}\right)+\gamma, \gamma \in F$. But since $q$ and $P^{\prime}\left(f_{1}, \cdots, f_{k}\right)$ are in $A$ it follows again from the direct sum property of $\bar{A}$ that $\gamma=0$ and hence $f_{1}, \cdots, f_{k}$ generate $A$, and the proof is complete.

Both the problem above and the method of solution were suggested by reading the paper $A$ note on finite ring extensions by Artin and Tate (Japanese Journal of Mathematics vol. 3 (1951) pp. 74-77) and I am indebted to Dr. Tate for suggestions which lead to simplification of my original proof.

BROWN UNIVERSITY 